\title{
Comprendre le travail de mise en œuvre de la réforme des études en Masso-Kinésithérapie (2015) : le cas d'un institut de formation
}

Understanding the work done to implement the Physiotherapy studies reform

(2015): the case of a training institute

Éric Maleyrot, Thérèse Perez-Roux, Charlotte Pourcelot et Pierre Hébrard

\section{OpenEdition}

Journals

Édition électronique

URL : http://journals.openedition.org/activites/3853

DOI : 10.4000/activites.3853

ISSN : $1765-2723$

Éditeur

ARPACT - Association Recherches et Pratiques sur les ACTivités

Référence électronique

Éric Maleyrot, Thérèse Perez-Roux, Charlotte Pourcelot et Pierre Hébrard, « Comprendre le travail de mise en œuvre de la réforme des études en Masso-Kinésithérapie (2015) : le cas d'un institut de formation », Activités [En ligne], 16-1 | 2019, mis en ligne le 15 avril 2019, consulté le 09 août 2019 URL : http://journals.openedition.org/activites/3853 ; DOI : 10.4000/activites.3853

Ce document a été généré automatiquement le 9 août 2019

\section{c)}

Activités est mis à disposition selon les termes de la licence Creative Commons Attribution - Pas d'Utilisation Commerciale - Pas de Modification 4.0 International. 


\title{
Comprendre le travail de mise en œuvre de la réforme des études en Masso-Kinésithérapie (2015) : le cas d'un institut de formation
}

\author{
Understanding the work done to implement the Physiotherapy studies reform \\ (2015): the case of a training institute
}

Éric Maleyrot, Thérèse Perez-Roux, Charlotte Pourcelot et Pierre Hébrard

\section{NOTE DE L'ÉDITEUR}

Article soumis le 3 juin 2018, accepté le 15 février 2019

Comprendre le travail ou plus exactement comprendre la transformation « du travail sur autrui » (Dubet, 2002), où la dimension relationnelle est essentielle, constitue aujourd'hui une entreprise complexe. En effet, le travail se déploie dans le cadre d'un système de normes organisationnelles, techniques, de qualité en constante évolution, cherchant à s'ajuster aux contraintes d'un marché de plus en plus ouvert à la concurrence. Les réformes institutionnelles participent de ces évolutions. Depuis une dizaine d'années, celles-ci traversent les formations des métiers adressés à autrui (enseignement, éducation, santé, travail social). Ainsi, dans le domaine de la santé, la formation initiale des personnels paramédicaux a connu une succession de réformes des études, d'abord en soins infirmiers (2009), en ergothérapie (2010), puis en pédicurie-podologie (2012), et, dernièrement, en masso-kinésithérapie (2015).

2 Ces réformes désirées ou subies par les différents acteurs de la formation (enseignants, formateurs, responsables de formation) engendrent inévitablement divers changements et redéfinissent le travail lui-même. Elles amènent à interroger non seulement les 
nouvelles normes du travail, inscrites dans les textes institutionnels, mais également l'appropriation de ces normes par les acteurs eux-mêmes.

3 Après avoir étudié les incidences des réformes $(2010,2013)$ de la formation des enseignants sur les formateurs (Maleyrot, 2012 ; Perez-Roux, 2012), de la formation des infirmières et des travailleurs sociaux (Hébard, 2011, 2013a, 2013b), il s'agit ici, à partir des dimensions prescrites et explicites de la réforme 2015 de la formation initiale des Masseurs-Kinésithérapeutes (MK), de tenter de comprendre les traductions (Akrich, Callon, \& Latour, 2006) des acteurs de la formation pour s'adapter aux évolutions et trouver du sens au travail qu'ils font. Plus précisément, l'étude s'intéresse à l'équipe de direction et aux formateurs d'un Institut de Formation de Masso-Kinésithérapie (IFMK) confrontés à la réforme des études dans les premiers moments de son déploiement. Elle vise à dégager les processus d'appropriation de la réforme, comprise par ces acteurs comme un nouveau modèle de formation initiale qui oriente leur travail dans un double mouvement d'universitarisation et de professionnalisation et qui, par voie de conséquence, amène à des transformations du métier de formateur.

4 Cet article comporte six parties. La première décrit les changements apportés par la réforme 2015 des études de MK à travers une analyse comparative des textes régissant ce dispositif de formation: le programme de 1989 et les référentiels de 2015. En deuxième lieu, sont exposées les notions de professionnalisation et d'universitarisation qui composent le corpus théorique. Une troisième partie définit la problématique développée dans le cadre de la sociologie de la traduction puis la méthodologie plurielle utilisée. La présentation des résultats de la recherche, en quatrième lieu, se compose de trois points. Les premier et second points présentent respectivement les préoccupations de l'équipe de direction puis des formateurs de l'école la première année de mise en place de la réforme. Le troisième point s'attarde à montrer l'évolution de la traduction locale de la réforme à travers le point de vue de l'équipe de direction la seconde année. Une cinquième partie, avant la conclusion, met en relief les tensions prégnantes et les décalages au cœur des transformations du métier d'enseignant/formateur en masso-kinésithérapie.

\section{Mise en contexte}

5 Comprendre le travail peut consister à l'analyser dans ses dimensions prescrites et tenter de comprendre les logiques explicites et implicites des concepteurs et initiateurs de la réforme.

6 Cette réforme du dispositif de formation ${ }^{1}$ des MK mise en place en 2015 modifie le dispositif précédent datant de 1989. L'analyse documentaire des textes instituant ces deux dispositifs (décret du 5 septembre 1989, décret et arrêté du 2 septembre 2015) met en évidence deux conceptions différentes de la formation. La première repose essentiellement sur un programme d'enseignement, c'est-à-dire une liste de contenus, de connaissances à acquérir, alors que la seconde est fondée sur une approche par les compétences. Nous avons étudié d'autres dispositifs de formation, mis en place dans les secteurs de la santé et du travail social au cours des années 2000 et se référant à une approche par les compétences (Hébrard, 2013b). Nous avions à cette occasion rappelé l'origine de cette conception de l'enseignement et de la formation professionnelle, issue du courant behavioriste (Elias, \& Merriam, 1980/1983 ; Tremblay, 1990). Jonnaert (2002) en propose un modèle "socioconstructif et interactif ", sans surmonter réellement les paradoxes et les ambigüités de cette approche par les compétences, tel qu'elle est 
notamment présentée et mise en œuvre en France dans la formation aux métiers de la santé (Coudray, \& Gay, 2009 ; Hébrard, 2013a). Plusieurs auteurs en ont montré les risques et les limites que ce soit dans le domaine de l'enseignement ou dans celui de la formation professionnelle (Allal, 2002 ; Crahay, 2006; Haberey, \& Heeb, 2015; Ollagnier, 2002 ; Stroobants, 2002). L'analyse qui suit vise à caractériser l'approche par les compétences préconisée par le nouveau dispositif de formation et ce qui le distingue du programme antérieur ${ }^{2}$.

\subsection{Le décret de septembre 1989 : une approche centrée sur les contenus}

7 Le texte de 1989 est un document d'une vingtaine de pages constitué d'un tableau intitulé «Programme horaire des enseignements théoriques et pratiques », présentant les quatre modules de la première année, les douze modules des deuxième et troisième années. Le reste du document présente le " programme des enseignements théoriques », c'est-à-dire les contenus détaillés des enseignements de chaque module, précédés, pour certains d'entre eux, des objectifs du module, définis en termes d'acquisition de connaissances théoriques, de techniques, de gestes thérapeutiques. La dernière page du document présente les objectifs et la durée des stages pratiques.

8 Ce qui est dominant dans la conception de ce dispositif de formation, ce sont les contenus à acquérir, les connaissances théoriques et techniques enseignées dans les cours théoriques, les travaux dirigés et travaux pratiques. Par ailleurs, une grande liberté est laissée aux instituts de formation quant à l'agencement fonctionnel du dispositif puisque les directeurs fixent le cadre général et les modalités de l'enseignement des modules des trois années d'études, chaque enseignant restant maître des contenus traités. Enfin, la notion de compétence est complètement absente de ce document.

\subsection{Les modifications du contexte institutionnel apportées par la réforme des études de MK de 2015}

9 Le texte instituant la réforme 2015 de cette formation est beaucoup plus volumineux. Il est composé d'un arrêté de dix pages accompagné de cinq annexes de 124 pages.

Ce qu'il faut retenir, dans le contenu de l'arrêté, c'est tout d'abord l'intégration de cette formation dans le cursus $\mathrm{LMD}^{3}$ et l'obligation de passer une convention entre chaque centre de formation délivrant cette formation, une université disposant d'une composante santé et le conseil régional (article 1). L'article 2 modifie la durée de la formation: celle-ci compte désormais quatre années au lieu de trois et elle est précédée d'une année universitaire validée (au total cinq années d'enseignement supérieur). L'article 3 mentionne que les étudiants sont inscrits à l'université et à l'institut de formation. Dès les trois premiers articles du décret «l'universitarisation" de cette formation est donc mise en avant.

11 D'autre part, cette réforme se situe clairement dans le courant de l'approche par les compétences. Celle-ci consiste à concevoir le dispositif de formation à partir du référentiel des activités du métier concerné, sur la base duquel sont élaborés le référentiel de compétences, le référentiel de formation et le référentiel de certification (Coudray, \& Gay, 2009). La notion de compétence, selon le répertoire des métiers de la 
Fonction Publique Hospitalière, est définie comme «la maitrise d'un savoir-faire opérationnel relatif aux activités d'une situation déterminée requérant des connaissances et des comportements " (cité par Coudray, p. 41). Cette définition est proche de celle de l'AFNOR: "capacité éprouvée à mettre en œuvre des connaissances, savoir-faire et comportements en situation d'action, dans un contexte donné » (2007, p. 6).

Dans le dispositif qui nous intéresse, chaque compétence est décrite avec un ou deux verbes d'action et elle définit une fonction en relation avec les activités du MK mobilisant différents savoirs (savoirs des sciences fondamentales, savoirs disciplinaires, savoir-faire associés d'éducation et de rééducation en masso-kinésithérapie) qui permettent d'accomplir efficacement et pertinemment une tâche. Cette compétence " est hétérogène par ses constituants et homogène par sa finalité technico-sociale » (Rey, 2006, p. 95).

Une analyse plus fine du dispositif de formation, à travers le contenu des cinq annexes de l'arrêté de 2015, permet de spécifier les caractéristiques de ce dispositif.

\subsection{Les annexes de l'arrêté de 2015 : un cadrage prescriptif fort}

L'annexe I intitulée «Référentiel des activités » définit les rôles et les missions des MK, leurs conditions d'exercice, et leur champ d'intervention. La liste des dix "activités principales » est suivie, pour chacune d'entre elles, d'une série "d'activités détaillées » (au total plus de 200)4. L'annexe II «Référentiel de compétences » contient une liste de onze compétences, puis, pour chaque compétence principale, une série de compétences détaillées (130 au total pour les onze compétences). Enfin, un tableau indique, pour chaque compétence trois à sept critères d'évaluation et, pour chaque critère, une liste d'indicateurs (au total plus de 300 indicateurs). L'annexe III définit la maquette de formation et l'annexe IV présente le référentiel de formation en détaillant d'abord sous forme de fiches les 32 Unités d'Enseignement (UE) puis en exposant les finalités, principes pédagogiques et modalités d'organisation de la formation. Il est à noter ici que l'essentiel de l'augmentation du volume horaire de la formation lié au passage de trois à quatre ans est constitué par des heures de «temps personnel». 1610 heures pour chaque cycle de deux ans y sont consacrées, soit la moitié du temps de formation en école. Par ailleurs, le mouvement d'universitarisation apparait encore lorsqu'il est écrit que « la formation doit également amener l'étudiant à concevoir et à réaliser des travaux de recherche pour permettre le développement de l'esprit scientifique, de la problématisation et de la réflexivité, transposables dans les pratiques professionnelles d'éducation et de soins $»^{5}$. Enfin l'annexe $\mathrm{V}$ fournit un exemplaire du portfolio composé de fiches d'objectifs, d'autoévaluation, d'évaluation des compétences, d'évaluation des stages.

Ce qui caractérise les outils contenus dans ces annexes c'est, d'une part, l'approche centrée sur les compétences avec son côté formel et ses longues listes d'activités et de compétences détaillées, de critères et d'indicateurs d'évaluation et, d'autre part, la coévaluation réalisée par l'étudiant en stage et le tuteur.

Cependant l'analyse que nous avons menée d'autres dispositifs privilégiant l'approche par les compétences en ingénierie de la formation (Hébrard, 2011, 2013a, 2013b) nous incite à penser que la complexité et la lourdeur de l'ensemble provoquent souvent des difficultés, notamment au niveau de l'évaluation de ces compétences pour les utilisateurs de ces outils. Si les concepteurs de la réforme de la formation initiale des MK mettent en avant, dans le paradigme du praticien réflexif (Schön, 1994), "un professionnel de santé autonome, responsable et réflexif, c'est-à-dire un praticien capable d'analyser toutes 
situations de santé, de prendre des décisions, en mesurant les limites de ses compétences ${ }^{6}$, il apparaît dans le même temps un dispositif fortement prescriptif, à travers ces référentiels et ces grilles d'évaluation découpant l'activité et les compétences en unités élémentaires et multipliant les critères et les indicateurs. Ce dispositif ne vientil pas en contradiction avec l'intention de former des professionnels autonomes et réflexifs? C'est en tout cas ce que soutiennent certains auteurs qui reprochent à l'approche par les compétences son aspect techniciste, voire behavioriste (Crahay, 2006 ; Elias, \& Merriam, 1980/1983 ; Stroobants, 2002) et sa difficulté à prendre en compte les dimensions relationnelles et sociales de l'activité (Carignan, \& Fourdignier, 2013; Hébrard, 2016).

Toujours est-il que la réforme de la formation initiale des MK, mise en place à la rentrée 2015, intègre une double logique de professionnalisation (Bourdoncle, 1991; Wittorski, 2008) et d'universitarisation (Bourdoncle, 2007).

\section{Corpus théorique}

Les deux notions de professionnalisation et d'universitarisation constituent le corpus théorique de cet article. Leur forte sollicitation dans les derniers textes institutionnels demande à ce que leurs fondements soient éclairés.

\subsection{La professionnalisation : un processus multidimensionnel}

19 Le terme de professionnalisation demeure fortement polysémique. Pour Bourdoncle (1991), la professionnalisation recouvre trois types de processus et trois résultats de ces processus. Tout d'abord, le "professionnisme » caractérise le résultat d'un processus de transformation de l'activité en profession autonome ayant un certain contrôle et monopole d'exercice, c'est-à-dire de professionnalisation statutaire par des négociations entre l'État, les associations professionnelles et les syndicats. «La professionnalisation désigne alors ce processus d'amélioration collective du statut social de l'activité » (Bourdoncle, 1991, p. 75-76). Dans le cas des MK, il pourrait se manifester par la valorisation de leurs savoirs médical et paramédical et la recherche d'une autonomisation en s'affranchissant de la tutelle des médecins.

Ensuite, la "professionnalité » désigne le résultat du "processus d'amélioration des capacités et de rationalisation des savoirs mis en œuvre dans l'exercice de la profession, ce qui entraîne une plus grande maîtrise et une plus grande efficacité individuelle et collective » (Ibid., p. 75). Ce processus peut être qualifié de développement professionnel. Dans le contexte ici de la formation initiale des MK, ce développement signifie l'augmentation des compétences professionnelles telles que prescrites dans le référentiel et par un allongement des études.

21 Enfin, le «professionnalisme» marque, à l'échelle de l'individu, un processus d'«adhésion à la rhétorique et aux normes établies collectivement» (Ibid., p. 76) par le groupe professionnel. Un individu fait preuve de professionnalisme lorsqu'il partage les valeurs et les normes explicites et implicites du milieu, respecte dans sa pratique des règles collectives, montre une conscience professionnelle et une exigence d'efficacité. La socialisation professionnelle constitue le processus conduisant à cet état. 
22 De son côté, Wittorski (2008) fait apparaître un autre sens qu'il désigne par la « professionnalisation-travail». C'est le sens utilisé par les organisations, lorsqu'elles développent la rhétorique de la flexibilité des personnes au travail pour leur plus grande efficacité. La mise en évidence de ce dernier sens est intéressante parce qu'elle met en relief « le débat faisant prévaloir, côté organisation, la logique compétence (proposée dans un souci d'accompagnement des évolutions du travail et, probablement aussi, pour répondre à un enjeu implicite de repositionnement du pouvoir dans les organisations au profit des directions d'entreprises) et, côté acteurs, la logique qualification (proposée et négociée dans un souci de mis en reconnaissance des professionalités à l'initiative des salariés, dans les organisations) » (Wittorski, 2008, p. 108).

Par ailleurs, ce débat soulève les enjeux du «décloisonnement des milieux de l'enseignement (notamment universitaires) et des milieux professionnels » (Ibid., p.110). Une mise en correspondance plus forte des formations et du travail dans les organisations est attendue avec «une intention de professionnalisation de la formation " (Ibid.), Wittorski parle alors de professionnalisation-formation", revenant au sens 2 de la professionnalisation de Bourdoncle. Il s'agit de proposer une nouvelle ingénierie de la formation, de repenser les dispositifs afin de favoriser, non seulement, l'insertion professionnelle, mais également, le développement de professionnels plus adaptables en fonction de l'évolution continue des organisations et technologies du travail. Les milieux de la formation sont amenés à s'intéresser à la dimension formative de l'activité en contexte professionnel et à (re)penser l'articulation ou le principe de l'alternance entre espace de formation et lieu de travail. Ils sont également amenés à mobiliser des outils de rationalisation discursive des pratiques permettant l'identification des principes organisateurs de celles-ci (Wittorski, 2003); en ce sens, l'analyse des pratiques ou l'écriture d'un mémoire professionnel visent à développer «la posture réflexive des formés ${ }^{7}$ » et donc leur capacité d'adaptation aux systèmes d'activité dans le champ du travail.

Éclairons maintenant le processus d'universitarisation de la formation.

\subsection{L'universitarisation de la formation : un processus complexe}

25 Historiquement, l'université se distingue par deux aspects que sont la division entre les savoirs du corps et de l'action d'une part, savoirs des mots et des écrits d'autre part (Bourdoncle, 2007). Selon cet auteur, « il y a universitarisation lorsque les institutions de transmission des savoirs d'un secteur professionnel, ces savoirs eux-mêmes et les formateurs qui les transmettent se trouvent en quelque sorte absorbés par l'université » (p. 138). Ainsi trois aspects concourent au processus d'universitarisation.

Le premier concerne « les institutions de formation d'origine [qui] disparaissent ou sont profondément transformées au profit des structures universitaires habituelles et de leurs modes ordinaires de fonctionnement " (Ibid., p. 139). Il semble que cela ne soit pas le cas des IFMK, qui, selon l'Arrêté du 2 septembre 2015, passent une convention avec une université disposant d'une composante santé. Les étudiants sont ainsi inscrits administrativement à l'IFMK et à l'université avec laquelle il a été conventionné et leur inscription pédagogique se fait au sein de l'institut. Le diplôme obtenu reste un Diplôme d'État de Masseur-Kinésithérapeute même si son obtention s'effectue par la validation des UE et des stages, sous forme de crédits européens accumulés tout au long des huit semestres de la formation. 

seulement transmis, mais aussi créés et accumulés selon les règles particulières de l'université faisant une large place à l'activité de recherche » (Ibid, p.138). Les instituts de formation et les écoles professionnelles sont habituellement plus utilisateurs et transmetteurs que créateurs de savoirs. L'université, liant enseignement et recherche, peut être à la fois créatrice de pratiques et de savoirs nouveaux, mais également conservatrice des savoirs et des pratiques anciennes. Cependant, ces savoirs ne sont pas transmis tels quels, l'universitarisation correspondrait à «un processus de disciplinarisation secondaire, reprenant les savoirs professionnels pour les reconstruire rigoureusement, selon le processus ordinaire de la constitution disciplinaire » (Ibid, p. 142). L'action formative consisterait, au contraire, à aboutir à une meilleure compréhension des pratiques et des terrains réels d'exercice, sans confondre différentes formes de rationalité ayant chacune leurs procédures de validation: la rationalité épistémique d'une part, la rationalité téléologique, d'autre part (Habermas, 1999). Cette dernière reposant sur les relations entre une intention d'agir, le choix réfléchi de moyens adaptés à l'objectif visé, condition de l'efficacité de l'action productive.

Enfin, un dernier aspect intéresse «les personnels de formation eux-mêmes [qui] se voient confrontés à un nouveau statut dominant, celui d'enseignant-chercheur, qui exige, pour y accéder, un doctorat » (Bourdoncle, 2007, p. 138). Les directions des institutions de formation professionnelle supérieure recrutent leurs formateurs sur la base de leur expérience dans la profession à enseigner et sur leurs qualités en tant que formateur, mais très peu sur des compétences en recherche. Bourdoncle (2007) évoque deux stratégies possibles pour associer les professionnels à l'enseignement universitaire. La première est celle du « double métier » où le professionnel du secteur est en même temps formateur des futurs professionnels. Cette solution autorise le maintien du contact du formateur avec la pratique professionnelle qu'il exerce à mi-temps et lui permet d'en saisir in vivo les évolutions. La seconde stratégie est celle du «temps plein ». Elle favorise l'universitarisation du formateur. Cette solution à l'avantage de permettre à ce dernier de consacrer complètement son activité professionnelle à la formation en s'appuyant de manière étroite sur des travaux de recherche ou même d'en réaliser, mais dans le même temps, l'éloigne de la pratique professionnelle d'origine.

Fort de ces deux notions, il est temps de présenter la problématique de cette étude et la méthodologie utilisée pour y répondre.

\section{La démarche de recherche}

Dans une première partie, nous présentons la problématique de cette étude. Celle-ci est développée dans le cadre de la sociologie de la traduction (Akrich, Callon, \& Latour, 2006) qui nous semble pertinente pour éclairer la façon dont l'équipe de direction et des enseignants de l'institut se sont saisis de la réforme. Nous abordons, dans une seconde partie, la méthodologie utilisée pour répondre à cette problématique.

\subsection{La problématique : une traduction à travers un réseau d'acteurs}

$31 \mathrm{Si}$, dans les textes de la réforme de 2015 , le processus d'universitarisation de la formation semble aller de pair avec celui de professionnalisation, comment ce double processus peut-il trouver traduction localement dans les IFMK?

Activités, 16-1 | 2019 

de direction des IFMK. Celles-ci en tant que cadres locaux sont chargées d'adapter aux contingences territoriales les prescriptions émanant de l'administration centrale ainsi que d'assurer l'effectivité de leur mise en œuvre et des résultats produits. La chaine de traduction - au sens que donnent à cette notion les spécialistes de la théorie de l'acteurréseau (Callon, 2006 ; Hassard, \& Law, 1999) - qui lie au centre les agents/acteurs locaux, suppose une suite d'adaptations ou d'ajustements à chaque étape et à chaque palier de son processus. Ce sont les équipes de direction qui sont le plus directement concernées par ce travail d'ajustement, en respect des grands objectifs fixés par la réforme des études. Leur proximité avec les personnels de l'établissement les conduit, dans un esprit de donnant-donnant, à recourir à une tactique qui s'apparente à celle de l'intéressement, notion qui chez Akrich, Callon et Latour (1991) renvoie à une interaction entre un intérêt individuel et une action collective a priori pour le mieux-être de l'un et de l'autre.

Se pose alors une première question relative à l'organisation des enseignements et de la collaboration entre les enseignants d'un même institut. Qu'ils soient concernés directement parce que formateurs à temps plein à l'IFMK, qu'ils le soient de manière plus lointaine parce qu'y exerçant ponctuellement ou encore parce que, principalement sur leur lieu d'exercice professionnel, ils accueillent les étudiants en stage, le couplage dans l'action des différents personnels de ces instituts constitue un des objectifs principaux pour l'atteinte collective des buts visés par la réforme. L'efficacité de l'action impulsée par le plus haut niveau de responsabilité, ici le Ministère de la Santé, dépend en effet fortement de la manière dont les traductions qui en sont opérées aux divers échelons de son organisation et de sa mise en œuvre sont couplées, c'est-à-dire en cohérence les unes avec les autres.

Une seconde question émerge quant à la professionnalité du MK à former, dans un métier où le rapport au corps est intrinsèque à leur travail. La précédente formation reposait sur l'acquisition de connaissances théoriques et pratiques visant essentiellement la maitrise des techniques et des gestes en masso-kinésiethérapie. Avec le double processus d'universitarisation-professionnalisation de la formation, l'hypothèse d'une certaine tension peut être posée, voire de débats au sein du corps professionnel entre ceux qui partagent la vision d'un développement professionnel par une rationalisation discursive de la pratique, davantage universitarisée, et ceux pour qui l'expérience sensible, éprouvée à travers la relation corporelle et relationnelle, reste première à la profession.

Dans ce moment de transition institutionnelle, notre étude vise à comprendre, à l'échelle d'un IFMK, les traductions (Akrich et al., 2006) opérées par les acteurs de la formation (équipe de direction, formateurs et tuteurs de stage).

Plus précisément, il s'agit d'analyser comment l'équipe de direction s'y prend pour surmonter les éventuelles tensions que nous avons soulevées et pour engager ses formateurs dans le nouveau dispositif de formation. Il s'agit aussi d'analyser les difficultés de couplage entre les acteurs dans la traduction de la réforme opérée par la direction. Enfin, il s'agit de repérer les éventuelles incidences sur la professionnalisation même des formateurs, chargés de l'implantation locale des orientations de la réforme.

\subsection{Une méthodologie qualitative à visée compréhensive}

37 Dans cette visée compréhensive, une méthodologie qualitative a été mise en œuvre. La démarche est à la fois située et longitudinale. 


\subsubsection{Une enquête située}

L'enquête s'est focalisée sur un IFMK situé en France métropolitaine, suite à la demande par l'équipe de direction d'un accompagnement, par une équipe de chercheurs en sciences de l'éducation, dans la mise en place de la réforme des études de MK de 2015.

Cet institut de formation a le statut juridique d'association loi 1901. À la rentrée 2015, il est dirigé par une nouvelle équipe de direction de deux personnes. Si le nouveau directeur connaît bien l'établissement pour y avoir enseigné pendant près de 20 ans et assuré des responsabilités pédagogiques, son directeur adjoint découvre la formation initiale des MK puisqu'il exerçait en tant que MK dans le monde sportif. Précisons que les deux directeurs sont inscrits dans un cursus universitaire en master de sciences de l'éducation durant cette année.

Le collectif de formateurs s'élève à une centaine de personnes. Pour la plupart, ce sont des MK professionnels exerçant en secteurs libéral ou public, mais interviennent également des médecins, des préparateurs physiques, des psychologues. Ils enseignent souvent dans leur spécialité professionnelle puisque, pour beaucoup, ils ont un parcours complémentaire au diplôme de MK (spécialisation, formation d'ostéopathe, etc.). L'établissement accueille 350 étudiants sur les trois années du cursus de formation (au moment de l'enquête, aucun étudiant n'est encore en $4^{e}$ année du nouveau cursus).

\subsubsection{Un recueil de données plurielles}

Parce que le milieu professionnel des MK était inconnu des chercheurs, et afin de repérer les interrelations in situ entre les acteurs de la formation, il a été mis en place une méthodologie d'enquête opérant une triangulation dans la collecte des données. Triangulation qui s'est effectuée, d'une part par le type de données plurielles: observations, entretiens et focus group (Kitzinger, 1995) et, d'autre part, par les informateurs multiples : équipe de direction, formateurs à l'IFMK et tuteurs de stage.

2 Tout d'abord, l'équipe de recherche a procédé, à partir d'une approche ethnographique par immersion dans la structure (Becker, 2002 ; Laplantine, 2006), à des observations à la fois flottantes et focalisées. Flottantes afin de saisir l'organisation locale du travail et les interrelations professionnelles, et focalisées lors de moments de présentation de la réforme et de réunions des régulations initiées par l'équipe de direction.

Parallèlement ont été menés trois focus group auprès de 12 formateurs volontaires (tuteurs de stage et enseignants à l'IFMK) pour aborder la « réception » de la réforme et ses effets sur la formation. Ils ont été réalisés tout au long de la première année de mise en place de la réforme, en janvier, mars et mai 2016. Pour chacun d'entre eux, ils ont regroupé six à huit formateurs présentant des caractéristiques contrastées : hommes/ femmes, emploi salarié/libéral, enseignants expérimentés/débutants, enseignants en centre/tuteurs de stage.

Enfin, l'enquête a donné lieu à des entretiens semi-directifs compréhensifs (Kaufmann, 1996) auprès de la direction de l'école, afin de rendre compte de l'évolution des problématiques rencontrées et de leurs résolutions dans le cadre de la traduction de la réforme au niveau local. Une première interview d'une heure a été réalisée auprès du directeur en janvier 2016. Un second entretien de plus de deux heures s'est tenu avec le 
directeur et son adjoint en novembre 2016, soit un an après la mise en place de la réforme.

\subsubsection{Une analyse thématique longitudinale} trois points. Le premier focalise sur le centre du réseau d'acteurs avec les problématiques rencontrées par le directeur la première année de la réforme. Le second point rapporte celles des formateurs volontaires exprimées à trois moments de la première année. Enfin, les difficultés et les avancées dans la traduction de la réforme au niveau local par l'équipe de direction en début de seconde année sont mises en relief dans un dernier point.

\subsection{De multiples chantiers à ouvrir pour le directeur la première année de la réforme}

Nouvellement nommé, le directeur de l'IFMK met en exergue les difficultés rencontrées et les réorganisations qu'il cherche à initier depuis la rentrée 2015. 


\subsubsection{L'opportunité d'un recadrage et d'une nouvelle dynamique locale}

liberté totale et complète de l'enseignement, du volume horaire, du programme qu'ils réalisaient", le directeur souhaite un "changement d'orientation organisationnelle, structurelle et pédagogique de l'école ». Il essaie de combiner ou de s'appuyer sur deux bases, celle du « domaine de l'existant » et celle du « texte de 2015 » pour mettre en place "un cadre pédagogique plus dynamique ». En un certain sens, la réforme constitue une opportunité pour « un recadrage » de la répartition du volume horaire des enseignants dans une présentation universitaire des enseignements en UE inscrites dans le référentiel de 2015. La réforme lui permet d'aller plus loin encore "dans la professionnalisation future, c'est-à-dire la profession de demain » en légitimant le développement d'une posture réflexive de l'étudiant qui s'inscrit dans l'évolution du métier et positionne le MK à un plus haut niveau professionnel. Cependant la réforme s'applique, à la rentrée 2015, au public d'étudiants de $1^{\text {re }}$ année, mais elle ne concerne pas les étudiants de $2^{\mathrm{e}}$ et $3^{\mathrm{e}}$ années qui continuent leur formation sous l'ancienne réglementation de 1989 . Le directeur a donc à gérer de front deux dispositifs de formation différents. Loin de constituer une rupture avec ce qui existait, il se trouve dans une période de transition entre une professionnalisation en voie de disparition et une professionnalisation/ universitarisation en émergence.

\subsubsection{Un nécessaire travail de traduction et de communication}

51

Contrairement à d'autres établissements qui préparaient la réforme des études depuis 7-8 ans, l'ancienne direction n'a pas anticipé le changement. En conséquence, les personnels n'ont pas été informés des modalités contenues dans la réforme. Il n'y a pas d'entraide entre les instituts. D'une part, aucun accompagnement des directions n'est prévu par le Ministère de la Santé. Le directeur et son adjoint apparaissent finalement isolés et en première ligne pour traduire la réforme sur le terrain. D'autre part, un certain nombre d'enseignants et plus encore les tuteurs demeurent éloignés des enjeux de la réforme, ce qui fait dire au directeur que « derrière, ça ne suit pas ».

La direction est ainsi contrainte à un travail de communication auprès des enseignants et des tuteurs. Des réunions d'information et de régulation sont organisées à cette fin. Les premières questions abordées concernent le cadre réglementaire de la réforme des études : «Qu'est-ce que la réforme? Comment cela va se passer?» La direction est amenée à expliciter les changements dans la professionnalisation des étudiants et à justifier les orientations vers la recherche. C'est à ce moment qu'elle sollicite une équipe de recherche en sciences humaines pour avoir un retour dans sa traduction de la réforme. Elle fait alors intervenir des membres de cette équipe pour inciter certains enseignants à s'inscrire en master à l'université et se former aux sciences de l'éducation. Le but du directeur consiste à construire "une organisation différente au niveau pédagogique » avec une équipe "d'enseignants-référents » proche de la direction, formés au double processus de professionnalisation/universitarisation, ayant pour mission de « chapeauter» des UE. 


\subsubsection{La question de l'intéressement des tuteurs de stage} révèlent les représentations sur la réforme et les controverses que celle-ci suscite. Les participant.e.s se saisissent de la demande des chercheurs : « où en êtes-vous à ce jour sur /qu'avez-vous à dire à propos de/quels sont les points essentiels pour vous dans... la mise en œuvre de la réforme de la formation des MK? » De façon dominante, les participants orientent les échanges sur des aspects problématiques. Quelques voix discordantes, plus en phase avec la réforme, peinent à se faire entendre. Elles se précisent au cours de l'année. De la même façon, des thématiques plus prégnantes apparaissent au fil des trois focus group réalisés.

\subsubsection{L'entrée par la professionnalisation}

57 L'approche de la réforme de la formation est d'abord envisagée comme une opportunité en termes de positionnement dans le champ médical/paramédical et, plus largement, en termes de reconnaissance sociale. De forts enjeux de revalorisation du métier sont perceptibles. Derrière les évolutions d'une formation universitarisée et intégrant une démarche réflexive, orientée par la recherche, le rapport aux médecins semble pouvoir être revisité. Si auparavant, les masseurs-kinésithérapeutes avaient pour rôle "d'appliquer les prescriptions», ils voient dans cette réforme la possibilité de se repositionner dans la hiérarchie médicale/paramédicale: "maintenant, grâce à la réforme, on va travailler avec eux et non plus pour eux [les médecins] ». Les discours appuient une revendication sur l'autonomisation de la profession et, à terme, une revalorisation salariale à « bac +5 ». Les échanges indiquent aussi que les MK se situent 
" au-dessus » des infirmières, sans trop expliciter pourquoi. Se redéfinir dans le champ paramédical, c'est aussi clôturer l'espace d'intervention et pousser au-dehors les intrus qui viennent y prendre place. De possibles concurrents sont évoqués : les titulaires d'un master STAPS, option Activités Physiques Adaptées (APA), susceptibles de prendre en charge une partie de l'accompagnement rééducatif, lors du retour au domicile du patient. Le fait que ces personnels «APA » soient titulaires d'un master 2, alors que la réforme des MK ne donnera à terme (2019 pour la première promotion) que le niveau master 1 leur laisse craindre une forme de déclassement.

58 Émerge une rhétorique de professionnalisation, qui a pour but à la fois la reconnaissance et la défense du groupe professionnel. Les nouvelles conditions de professionnalisation (professionnisme chez Bourdoncle, 1991) passent par l'universitarisation et ce qu'elle suppose en termes de savoirs constitués à transmettre. En même temps, dans les focus group, s'exprime la crainte d'une universitarisation au détriment de la professionnalisation, une tension entre savoirs et savoir-faire : «on va former des kinés avec une grosse tête, mais qui n'auront pas de mains». Par ailleurs persiste la crainte d'une mainmise des médecins (et du CHU) sur l'activité de formation et une diminution des marges de manœuvre pour penser la formation, la recherche et les partenariats.

\subsubsection{Des repères pédagogiques bousculés}

59 Les échanges lors des focus group portent aussi sur les déstabilisations liées aux nouveaux modèles de formation mis en avant. Entre approches transmissives adossées aux « bonnes pratiques ", où l'expérience tient lieu de laissez-passer, et approche par problèmes, orientée vers et par la réflexivité des étudiants, se joue la question des contenus, des dispositifs pédagogiques pour développer la nouvelle professionnalité attendue du MK, de la posture de l'enseignant ou du tuteur de stage. Les débats renvoient de façon indirecte aux tensions entre enseigner-former, entre transmettre et faire construire (Fabre, 2015).

Des craintes sont exprimées quant à l'adhésion des étudiants, au manque de temps, au besoin de transmettre des pratiques efficientes. Les tuteurs rendent compte de décalages, notamment au niveau de la démarche réflexive et du suivi de mémoire, désormais plus orienté vers la recherche. Cette nouvelle orientation pose donc un problème de positionnement et peut renvoyer un sentiment d'incompétence.

61 Enfin, les participants font état de nombreux malentendus sur le mémoire: quelles exigences théoriques et méthodologiques? Quelle répartition des tâches entre tuteurs et enseignants? Quelle légitimité à assurer ce suivi si l'on n'a pas soi-même un ancrage direct dans la recherche?

\subsubsection{Des tensions fortes}

Plusieurs tensions sont repérables dans les échanges. Tout d'abord, les représentations que les formateurs se font des étudiants sont relativement problématiques. De nombreux étudiants n'ayant pas obtenu leur premier vœu d'orientation suite à leur classement en fin de Première Année Commune aux Études de Santé (PACES) risqueraient d'envisager le parcours MK avec un certain mépris, comme une sorte de déclassement. La possible dévalorisation de la filière MK est mal vécue par les participants aux focus group. Cette problématique, non directement liée à la réforme des études, suppose alors de se repositionner comme formateur, de montrer ses savoirs et savoir-faire ou, plus largement, sa légitimité. Les formateurs présents insistent sur l'importance de montrer 
aux étudiants l'intérêt du métier, ses exigences, mais aussi les ouvertures qu'il permet. En effet, l'orientation " par défaut » des étudiants, associée à une revalorisation des savoirs scientifiques, et des postures réflexives peut faire craindre un faible engagement dans le travail autour des savoir-faire ou un rapport assez distancié à l'apprentissage des gestes professionnels. Cette éventualité rend compte, à mots couverts, d'une mise en danger des formateurs, pris au piège d'une nouvelle exigence de professionnalisation (par des étudiants et par leur direction) dont ils ne saisissent pas précisément les contours.

Une autre tension se joue entre théorie et pratique, à travers l'articulation complexe des contenus proposés à l'IFMK et ce qui se fait, se comprend, s'apprend sur le lieu de stage. Comment créer du lien entre ces deux espaces relativement disjoints? Comment articuler les regards des différents intervenants qui n'ont pas les mêmes contraintes, en vue de professionnaliser les étudiants? Comment adapter les contenus et méthodes lorsque les durées et les périodes de stage bousculent tous les repères antérieurs? Comment s'assurer du suivi sur le terrain et de l'articulation cohérente des attendus et du prescrit?

Par ailleurs, les échanges se focalisent sur l'évaluation et ses enjeux. Entrer dans une démarche réflexive, mettre en œuvre un dispositif innovant, c'est ouvrir de nouveaux espaces dans lesquels l'évaluation sommative peut poser problème. Comment par ailleurs évaluer les compétences d'un professionnel avec un mémoire? Quelle place prennent les tuteurs, les pratiques, la relation au patient ? De fait, si la réingénierie a été impulsée par la direction, les formateurs entrevoient les questions d'évaluation à venir, dans des dispositifs encore mal maîtrisés.

Une dernière tension se dessine au niveau des écarts entre experts et novices, entre MK généralistes et MK spécialistes. La réforme oblige en quelque sorte les professionnels à se questionner sur les compétences attendues et sur leur mode de construction en formation, sur la place des stages dans la découverte des pathologies, des patients, des spécificités et des milieux de la masso-kinésithérapie. Sur ce point, les avis divergent entre les participants, selon leur propre professionnalisation et leur positionnement dans le réseau des MK.

\subsection{Une direction en plein travail de traduction de la réforme la seconde année}

66 Ce second entretien avec la direction se déroule avec la participation du directeur et de son adjoint. Ceux-ci sont amenés, au début de cette seconde année universitaire à expliquer ce qu'ils ont mis et tentent de mettre en place dans l'école par rapport à la réforme.

\subsubsection{La mise en œuvre de dispositifs réflexifs jugés innovants}

Les deux directeurs ont créé un dispositif innovant de mise en situation professionnelle simulée qu'ils ont appelé «Groupe d'entrainement à l'analyse des pratiques rééducatives " (GEAPR) (Teisseire, Rouvière, Boussagol, \& Perez-Roux, 2018). Ils ont développé des projets, autour de groupes de recherche documentaire, sur des pathologies avec présentation orale et supports de communication tels que les posters. "Jamais on n'a autant axé la formation sur la partie de réflexion, de recherche, d'analyse bibliographique, de construction des connaissances que maintenant ». La direction est « en ébullition réflexive » pour faire évoluer les TD de manipulation : «On lance plusieurs 
modes différents d'enseignement, on est dans le modèle du GEAPR aujourd'hui sur au moins quatre thématiques de travail lancées sur deux promotions. On a l'expérience de l'année dernière, mais on innove aussi sur d'autres plans». «Ce qu'on souhaiterait développer c'est une forme d'apprentissage » c'est-à-dire passer du TD traditionnel «je fais voir le mouvement, je leur explique, ils le refont, je corrige » à des TD nouveaux où les étudiants mobilisent leurs connaissances et construisent celles qui leur manquent.

Devant les multiples chantiers de travail, la direction souhaite se délester progressivement de son activité de formation en face à face pédagogique : « on veut faire moins d'enseignements, on veut faire moins de trucs, parce qu'on y perd trop de temps, mais peut-être qu'on va perdre des enseignants à cause de ça, parce que... on n'a personne qui soit réellement formé ».

\subsubsection{Une dévolution nécessitant un changement de posture}

Pour former ses enseignants, la direction initie les volontaires à l'animation des séances de GEAPR par monstration : « ces enseignants, on les prend avec nous sur le dispositif, et on essaie de les sensibiliser et de montrer la relation qu'on met en place et la position de l'enseignant par rapport à l'éducation des étudiants ». Deux problématiques émergent de cette intention de formation.

70 Tout d'abord, un débat apparaît entre les deux directeurs quant à la place de l'évaluation de la pratique des étudiants. Le directeur est favorable à une auto-confrontation ${ }^{8}$ initiée par l'enseignant après la séance de GEAPR pour une correction de la pratique des étudiants. "Quand ils viennent me voir en auto-confrontation, là, je détruis. À un moment ou à un autre, la pratique, il faut quelqu'un qui y aille (...). Donc je lui explique un petit peu les limites de ce qu'il est en train de voir, de dire et d'avoir proposé à un patient. L'objectif, c'est ça. » Le directeur adjoint, quant à lui, défend l'objectif d'une évaluation pendant et après le GEAPR : « on va avoir le résultat qu'on recherche, c'est-à-dire l'autoévaluation, c'est-à-dire l'auto-critique, c'est-à-dire l'analyse des étudiants » et l'autoconfrontation post séance du GEAPR est intéressante pour une réassurance de l'étudiant sur sa pratique. "Quand on voit un étudiant qui a été en difficulté, qui l'exprime et qui sur le fond n'a pas été si mauvais que ça, on revient avec lui, on trouve que c'est intéressant aussi de lui proposer de revoir son activité ».

71 Par ailleurs, les directeurs expriment la difficulté à passer la main aux enseignants. Ils craignent que ceux-ci prennent « à leur initiative spontanée de refaire de l'enseignement dans ce processus» et de voir le dispositif dénaturé et "évoluer dans une autre orientation que celle qu'on a voulu faire ». Au-delà de l'intéressement des enseignants par la direction c'est, plus fondamentalement, la transformation de la posture $^{9}$ des enseignants qui est demandée. Ceux-ci doivent donc opérer un double déplacement. D'une part, il leur faut passer d'une posture de transmission par monstration-application d'un geste ou par exposition d'une pathologie à une posture de mise en réflexion des étudiants, c'est-à-dire « être plus des modérateurs dans la réflexion de nos étudiants que de dire : tu as fait ça, il faut que tu fasses ça ». D'autre part, ils sont incités à quitter des enseignements de leur domaine d'expertise professionnelle de MK, parce qu' « il va falloir à un moment qu'ils sacrifient quelque chose " pour aller enseigner dans des domaines qu'ils ne maîtrisent pas autant.

72 Ces deux problématiques montrent une traduction délicate de l'esprit de la réforme. Elle soulève, non seulement la place de l'évaluation au regard de la réflexivité recherchée et 
donc de la professionnalité attendue du futur MK, mais également la question de la transformation de la posture de l'enseignant investi dans la formation initiale en MK.

\subsubsection{Une avancée à deux vitesses} traduction asynchrone de la réforme au niveau local. Le second discute de la professionnalisation des formateurs de l'IFMK. Enfin, le dernier point s'attarde sur la question de l'évaluation des compétences des étudiants comme indicatrice d'une universitarisation-professionnalisation en tension.

\subsection{Une traduction asynchrone de la réforme} asynchrone de la réforme. Le réseau des acteurs n'est pas touché au même rythme ni avec la même intensité par la mise en œuvre au niveau local.

78 Une asynchronie apparaît à travers les thématiques abordées par la direction et les enseignants aux mêmes moments. Les discours de la direction et des formateurs révèlent un décalage des préoccupations. Dès la première année, la direction s'attache à opérationnaliser dans les UE les dimensions de réflexivité et de recherche préconisées par 
la réforme et à enrôler les personnels dans cette réorientation de la formation. De leur côté, les formateurs prennent connaissance des textes de la réforme qui n'a pas été anticipée par l'ancienne direction. Leurs soucis portent d'abord sur la professionnalisation de la profession (Bourdoncle, 1991; Wittorski, 2008) avec la satisfaction d'une reconnaissance de la profession par une certaine universitarisation. Parallèlement, les différents référentiels, constituant des outils de normalisation (Chauvigné, 2010), soulèvent chez les formateurs non seulement les craintes d'une universitarisation des savoirs professionnels au détriment de la maîtrise des gestes du métier, mais également des tensions entre théorie et pratique avec des conséquences pédagogiques sur leur travail de formateur.

79 Le décalage semble se prolonger la seconde année puisqu'une communication auprès des formateurs est rendue nécessaire à la fois pour informer des enjeux locaux de la réforme, mais aussi pour les intéresser (Akrich et al., 1991) collectivement à s'investir dans les dispositifs réflexifs jugés innovants. Cependant, les nouveaux textes ne donnent pas encore lieu, pour les formateurs, à une « mise en débat des représentations que chacun possède du métier visé par la formation, des manières de faire et donc de faire faire par les apprenants » (Balas, 2013, p. 102).

80 C'est donc une avancée à deux vitesses qui est constatée. D'un côté la direction en ébullition réflexive et grisée par la réussite de leur nouvelle ingénierie auprès des étudiants n'arrive plus à assumer tous les temps de face à face pédagogique avec ces derniers. D'un autre côté, les formateurs sont encouragés à se former et à prendre en charge l'enseignement dans ces nouveaux dispositifs.

81 Cette asynchronie s'avère être également une asynchronie dans l'espace du réseau local. C'est bien la direction, centre du réseau, qui se trouve en première ligne pour traduire les textes de la réforme. S'agissant d'une nouvelle équipe de direction, cette traduction vient en rupture par rapport à la culture locale de formation, jusqu'ici à l'œuvre. Les textes de la réforme constituent à la fois l'opportunité d'un recadrage des routines de formation existantes, visant à stopper un certain laisser-aller dans l'organisation des enseignements sous l'ancienne direction, et l'occasion d'une nouvelle dynamique de formation.

82 Même si la direction s'attache à enrôler tous les personnels la première année, l'intéressement des tuteurs de stage, éloignés du centre du réseau, paraît une tâche complexe. Complexe parce qu'une visée de développement d'une dimension réflexive et de recherche chez les étudiants se heurterait, selon Olry (2003), à une double difficulté de la professionnalisation des MK. D'une part, « les protocoles de soins kinésithérapiques ne sont pas stabilisés et ne peuvent constituer un savoir de référence; celui-ci reste à élaborer. [D'autre part] la relation avec le patient, comme l'identification et la sélection d'indices perceptifs sur le corps du patient relèvent de compétences implicites, incorporées par les professionnels » (p. 103). D'ailleurs, il semble bien que la direction focalise, la seconde année, sur les acteurs du réseau les plus proches, les enseignantsréférents notamment, n'évoquant plus les tuteurs de stage.

83 Le défi de rendre cohérente l'action des uns à l'IFMK et des autres sur les terrains d'exercice est grand, et l'alignement des acteurs du réseau n'est pas encore réalisé la seconde année de mise en œuvre de la réforme. Ce couplage ne semble pas être une simple adaptation ou ajustement des différents formateurs du réseau, mais demande un véritable travail de transformation de leur action de formation. 


\subsection{Une professionnalisation secondaire des formateurs à l'IFMK en question} ces formateurs (Wittorski, 2008). Par la réforme de la formation traduite par la direction, ils sont conduits, soit à quitter l'IFMK, soit à opérer un changement de posture. Rares sont ceux qui abandonnent - car être formateur constitue une reconnaissance par la profession - mais rester suppose de passer d'un rôle d'enseignant - c'est ainsi qu'ils se présentent - à une fonction de formateur. Centrés au préalable sur la transmission par monstration - application d'un geste ou d'explication d'une pathologie fondée par l'expérience acquise dans un milieu où « la pratique kinésithérapique mobilise un couple vertueux entre conformisme procédural et aménagements personnels des techniques » (Olry, 2003, p. 41) -, ils ont désormais à opérer un déplacement pour se focaliser sur le développement des compétences professionnelles, telles qu'elles sont prescrites dans le référentiel de compétences. Ce développement passe par l'entrainement à la réflexivité, l'initiation et l'accompagnement à/par la recherche, la communication et la discussion des travaux réalisés par les étudiants.

Or, ce déplacement n'est pas une simple adaptation aux contraintes de la réforme. Il oblige à une véritable transformation de son rapport au savoir, rapport que charlot (1990) désigne comme « rapport à l'apprendre ». En effet, la logique de l'enseignement est celle des discours constitués en systèmes et organisés autour de cohérences propres transmis 
de façon la plus claire ou la plus transparente possible. L'objectif visé par l'enseignant MK reste le savoir de/sur la pratique décontextualisé, censé « dire » le vrai dans le monde de la masso-kinésithérapie, même si le travail kinésithérapique relève avant tout d'un corps à corps avec le patient et d'une collaboration dans le dialogue thérapeutique (Olry, 2003). La logique de la formation s'avère tout à fait différente. Elle est celle des pratiques finalisées et contextualisées. Elle se centre sur l'individu et demande de l'accompagner dans le développement de compétences professionnelles à partir de savoirs hétérogènes, aussi bien issus de la pratique en masso-kinésithérapie que des disciplines médicales et de la recherche en santé ou en sciences humaines et sociales.

89 Sans leur demander de déposer leur expertise à la porte de l'IFMK, les enseignants ont conscience qu'ils ont à dépasser leur professionnalisation primaire dans le monde des MK pour opérer une professionnalisation secondaire de formateur en formation initiale. Cette attente d'une nouvelle professionnalisation, perceptible à travers leur inconfort actuel sur leur légitimité, a aussi à voir avec une certaine transformation identitaire (Perez-Roux, 2010). Elle pose en tout cas de façon cruciale la question de leur formation. Car aucun accompagnement n'est prévu en direction des enseignants pour une appropriation de la réforme, tant comme moyen de revitaliser leur propre professionnalisation que pour « relancer les débats d'écoles entre gens de métier et les controverses professionnelles et ainsi fabriquer un cadre favorable au développement individuel et collectif des professionnels concernés » (Balas, 2013, p. 94). Ceux-ci sont censés pouvoir mettre en œuvre ses orientations et des démarches ad hoc pour la construction des savoirs et compétences attendus chez les étudiants.

Il est alors aisé de comprendre que la direction s'entoure d'une équipe de chercheurs en sciences de l'éducation et incite fortement ses enseignants à s'inscrire en master dans cette discipline.

\subsection{L'évaluation : un indicateur d'une universitarisation- professionnalisation en tension}

91 La question de l'évaluation des compétences des étudiants souligne une universitarisation-professionnalisation en forte tension. Cette tension transparaît à travers plusieurs objets de la formation.

92 Tout d'abord au niveau du mémoire, les étudiants se dirigent désormais vers des recherches à orientation scientifique plus que professionnelle. L'entrée dans ce travail se fait par un thème plutôt que par un cas clinique. Les formateurs interrogés expriment ne pas être préparés à diriger ce type de mémoire. Si certains d'entre eux disent s'autoformer sur le tas et retirer un enrichissement par l'accompagnement des étudiants, on perçoit clairement une rupture dans les manières de faire jusque-là en vigueur. Les tuteurs notamment déclarent que lorsque les étudiants s'engagent dans la recherche, il est difficile d'exploiter leurs travaux pour retourner sur un cas clinique. En effet, « de multiples ouvrages existent qui décrivent, souvent sous forme de cas cliniques, des problèmes traitables par des protocoles constitués [mais] l'usage de ces formalisations se forge à l'épreuve du patient dans une sorte de logique d'exercice et constitue un savoir intermédiaire néanmoins explicitable»(Olry, 2007, p. 41). Estimant ne plus être véritablement légitimes pour assurer ce suivi en raison d'un faible ancrage dans la recherche, les tuteurs expriment une tension entre le sentiment de ne plus savoir ce qu'il faut faire et l'évaluation des savoirs dans la prise en charge des patients. Ils saisissent mal 
la place importante donnée à ce mémoire universitaire, mais surtout la manière d'évaluer, par les critères retenus, les compétences d'un professionnel qui, pour eux, reste un praticien.

Les stages constituent un autre point de tension. Ils ont une importance accrue pour la validation des compétences. La réforme confie ainsi un rôle fort aux tuteurs de stage qui ont la responsabilité de contribuer au développement des compétences des étudiants et d'évaluer leurs acquisitions pratiques en se servant du portfolio et des grilles d'évaluation. Or, les discours des tuteurs de stage montrent une préoccupation forte sur la validation des stages et laissent apparaitre un énorme décalage entre la réalité des pratiques et les attendus de la réforme. Ils s'interrogent sur la définition des objectifs de stage et le contenu des documents de liaison entre le lieu de stage et l'IFMK. Il semble bien qu'ils considèrent avant tout dans le stage «l'accomplissement de l'action comme une authentique praxis et non pas comme l'exécution d'instructions, l'application de plans ou la mise en œuvre en résultat d'un calcul d'une délibération » (Olry, 2003, p. 38). Là encore une tension émerge entre ce qu'ils perçoivent comme une dérive intellectuelle (démarche réflexive de résolution de problème soutenue par les recherches scientifiques) au détriment des savoir-être et savoir-faire attachés aux champs cliniques et aux gestes professionnels. C'est fondamentalement le rôle du tuteur de stage qui est interrogé. Pour un certain nombre d'entre eux, l'évaluation des études ne rend pas suffisamment compte de la pratique. Ainsi, dans certains lieux de stage, des mises en situations professionnelles sont réintroduites, pour participer à la validation du stage de l'étudiant et cela, malgré leur aspect chronophage et l'organisation importante qu'elles supposent pour la structure d'accueil.

Enfin, une troisième tension est également visible au niveau même de la direction de l'IFMK par le désaccord relatif à l'évaluation des étudiants dans le dispositif du GEAPR. Il s'agit pour le directeur de développer in fine des compétences individuelles relatives au procès de travail, c'est-à-dire mobilisées avec efficacité et sureté, en interaction avec les caractéristiques physiques de l'environnement. De son côté, le directeur adjoint privilégie une auto-évaluation collective formative et réflexive par les pairs, c'est-à-dire une logique de réflexion sur et pour l'action conduisant à produire des compétences de processus (Wittorski, 1998) au sens de connaissances formalisées, disciplinées sur les savoirs professionnels mobilisables en situation. Ce débat, circonscrit à la seule direction, peut apparaître comme ce que Balas (2013) appelle « un dilemme historique de métier ", c'est-à-dire une situation constitutive du métier de formateur où le choix entre deux voies reste insatisfaisant. Ici, ces deux conceptions donnent à voir deux voies d'accès à la responsabilité médicale qui seraient complémentaires pour le professionnel MK.

\section{Conclusion}

Notre objectif visait à comprendre les traductions opérées par des acteurs chargés de la mise en œuvre de la réforme 2015 des études de MK dans un institut de formation pour s'adapter aux évolutions et trouver du sens au travail qu'ils font.

Si cette étude s'attache à un seul IFMK parmi les 47 implantés sur le territoire métropolitain, si elle analyse les deux premières années de mise en place de la réforme en interrogeant les directeurs et un nombre limité d'acteurs du réseau local (enseignants et tuteurs de stage) sur leurs activités de formation, elle permet néanmoins de dégager les problématiques qu'ils rencontrent dans ce moment de transition institutionnelle. Trois 
niveaux du processus de traduction ont été mis en relief et sont susceptibles d'aider les acteurs à comprendre la réorientation de leur travail.

À un niveau macro, la traduction de la réforme par les acteurs montre une asynchronie dans l'espace du réseau local. Tel un objet touchant la surface de l'eau et produisant un train d'ondes transversales circulaire autour de l'impact, la réforme touche d'abord la direction, puis les formateurs proches intervenant à l'IFMK et enfin les tuteurs de stage sur les lieux de travail. L'interprétation des textes de la réforme au prisme du contexte local est d'abord développée par la direction avant que celle-ci cherche à intéresser les enseignants-référents puis les formateurs plus éloignés.

À un niveau méso, les tensions chez les formateurs révèlent un mouvement d'universitarisation-professionnalisation ambivalent. Ceux-ci sont tiraillés entre deux orientations: d'une part, la satisfaction d'une élévation vers des savoirs théoriques, soutenus par des apports scientifiques, rendant possible une autonomisation de la profession par rapport à la tutelle des médecins; d'autre part la motivation à transmettre les gestes sûrs, efficaces et à développer les compétences comprises comme la maitrise d'un savoir-faire requérant des connaissances et des comportements mobilisables immédiatement en situation de travail.

Enfin, à un niveau micro, la focalisation sur les formateurs intervenant à l'IFMK met en lumière leur professionnalisation secondaire, rendue nécessaire par le fait des nouvelles orientations de la réforme. Mais traduites exclusivement par la direction en des dispositifs innovants ou en modalités d'enseignement repensées, apparaît également une absence de dialogues et de débat entre formateurs sur l'objet même de leurs pratiques didactiques et pédagogiques. Difficile alors de passer "d'une logique de "formateurtransmetteur" d'un métier prédéfini à une logique de "formateur co-concepteur" de la cible professionnelle visée. » (Balas, 2013, p. 104).

100 Si ces trois niveaux interdépendants dévoilent une certaine épaisseur dans la complexité du travail de ces acteurs de la formation, il est à noter que les problématiques évoquées concernent l'activité de travail déclaré, les première et seconde années de mise en place de cette réforme. C'est donc une recherche qui, s'inscrivant dans une dynamique de changement du travail de ces acteurs, appelle à être poursuivie au sein du même IFMK et, plus largement, à s'ouvrir à d'autres IFMK.

101 Enfin, il demeure intrinsèquement une difficulté pour le chercheur à comprendre le travail, car les problématiques bougent sans cesse à des rythmes différents, modifiant les périmètres d'action de chacun des acteurs, selon sa position dans le réseau. Cette mobilité ne va pas sans questionner le chercheur sur l'utilité de ses travaux lorsqu'il doit rendre compte aux acteurs eux-mêmes de ses résultats, en se confrontant à des temporalités différentes. 


\section{BIBLIOGRAPHIE}

AFNOR (2007). Rapport du groupe de réflexion " compétence », COS 12. La Plaine Saint Denis : Association Française de Normalisation.

Akrich, M., Callon, M., \& Latour, B. (1991). L'art de l'intéressement. In D. Vinck (Ed.), Gestion de la recherche. Nouveaux problèmes, nouveaux outils (p. 27-52). Bruxelles : De Boeck Université.

Akrich, M., Callon, M., \& Latour, B. (2006). Sociologie de la traduction. Textes fondateurs. Paris : Presses des Mines.

Allal, L. (2002). Acquisition et évaluation des compétences en situation scolaire. In J. Dolz et E. Ollagnier (Eds.), L'énigme de la compétence en éducation (p. 77-94). Bruxelles : De Boeck Université.

Balas, S. (2013). De l'usage dénormalisant des référentiels. Un exemple pour la formation des masseurs-kinésithérapeutes. TransFormations, 9, 89-109.

Balas, S. (2016). Comment concevoir des référentiels de diplômes professionnels sans renoncer au travail réel ? Activités [En ligne], 13(2). URL : http://activites.revues.org/2889

Becker, H.S. (2002). Les ficelles du métier. Comment conduire sa recherche en sciences sociales. Paris : La Découverte.

Bourdoncle, R. (1991). La professionnalisation des enseignants : analyses sociologiques anglaises et américaines. Revue française de pédagogie, 94, 73-92.

Bourdoncle, R. (2007). Autour du mot : Universitarisation. Recherche et formation, 54, 135-149.

Carignan, L., \& Fourdrignier, M. (2013). Pratiques réflexives et référentiels de compétences dans les formations sociales. Québec, Presses Universitaires du Québec.

Callon, M. (2006). Les réseaux sociaux à l'aune de la théorie de l'acteur-réseau. Entretien avec Michel Ferrary. Sociologies pratiques, 13, 37-44.

Charlot, B. (1990). Enseigner, former : Logique des discours constitués et logique des pratiques. Recherche \& Formation, 8(1), 5-17.

Chauvigné, C. (2010). Les référentiels en formation. Recherche \& Formation, 64, 77-89.

Coudray, M-A., \& Gay, C. (2009). Le défi des compétences. Comprendre et mettre en œuvre la réforme des études infirmières. Paris : Masson.

Crahay, M. (2006). Dangers, incertitudes et incomplétude de la logique de la compétence en éducation, Revue Française de Pédagogie, 154, 97-110.

Demaizière, F. (2008). Le dispositif, un incontournable du moment. Apprentissage des langues et systèmes d'information et de communication, 11(2), 157-161.

Dubet, F. (2002). Le déclin de l'institution. Paris : Le seuil.

Elias, J. L., \& Merriam, S. (1980/1983). Penser l'éducation des adultes. Montréal : Guérin (Traduction française de Philosophical Foundations of Adult Education. New York : Robert Krieger Publishing Company).

Fabre, M. (2015). Penser la formation. Paris : Editions Fabert. 
Haberey, V., \& Heeb, J-L. (2015). Pour une critique de la compétence. La question du sujet et de la relation à l'autre. Paris : L'Harmattan.

Habermas, J. (1999, traduction française 2001). Vérité et justification. Paris : Gallimard.

Hassard, J., \& Law, J. (1999). Actor-Network Theory and After. Oxford, Royaume-Uni : Blackwell Publishers.

Hébrard, P. (2011). L'humanité comme compétence ? Une zone d'ombre dans la professionnalisation aux métiers de l'interaction avec autrui. Les Sciences de l'Éducation. Pour l'ère nouvelle, 44(2), 103-121.

Hébrard, P. (2013a). Ambiguities and paradoxes in a competence-based approach to vocational education and training in France. European Journal for Research on the Education and Learning of Adults, RELA, 4(2), 111-127. En ligne : http://www.rela.ep.liu.se/contents.asp?doi=10.3384/ rela.2000-7426.201342

Hébrard, P. (2013b). Quelle « approche par les compétences » et quels référentiels pour la formation professionnelle aux métiers de la relation humaine? Les Dossiers des Sciences de l'Éducation, 30, 17-34.

Hébrard, P. (2016). Formes et dispositifs de reliance pour la construction des identités professionnelles dans le secteur sanitaire et social. Phronesis, 5/1, 76-83.

Jonnaert, P. (2002). Compétences et socioconstructivisme. Un cadre théorique. Bruxelles : De Boeck.

Kaufmann, J.-C. (1996). L'entretien compréhensif. Paris : Nathan.

Kitzinger, J. (1995). Qualitative research: Introducing focus groups. BMJ, 311, 299-302.

Lameul, G. (2008). Les effets de l'usage des technologies d'information et de communication en formation d'enseignants sur la construction des postures professionnelles. Savoirs, 17, 71-94.

Laplantine, F. (2006). La description ethnographique. Paris : Armand Colin.

Maleyrot, E. (2012). Ruptures et transformations identitaires des maîtres formateurs face aux réformes de la formation des enseignants. Les sciences de l'éducation pour l'ère nouvelle, 45(3), 65-88.

Ministère des Affaires Sociales, de la Santé et des Droits des femmes, Arrêté du 2 septembre 2015 relatif au diplôme d'État de masseur-kinésithérapeute. BO Santé - Protection sociale - Solidarité ${ }^{\circ}$ 2015/8 du 15 septembre 2015. https://solidarites-sante.gouv.fr/fichiers/bo/2015/15-08/ ste_20150008_0000_p000.pdf

Ollagnier, E. (2002). Les pièges de la compétence en formation d'adultes. In J. Dolz \& E. Ollagnier (Eds.) L'énigme de la compétence en éducation (p. 183-202). Bruxelles : De Boeck Université.

Olry, P. (2003). Organisation du soin et développement des compétences. Contribution à la professionnalisation en masso-kinésithérapie. Les Sciences de l'éducation pour l'Ère nouvelle, 36(2), 91-105

Olry, P. (2007). Apprentissages informels dans l'activité : dispositif de participation et processus d'engagement du remplaçant en masso-kinésithérapie. Revue française de pédagogie, 160, 39-50

Perez-Roux, T. (2010). Identité professionnelle des formateurs d'enseignants de Lycée Professionnel en IUFM : enjeux et dilemmes à l'heure des réformes. Recherches en Éducation, 8, 8-49.

Perez-Roux, T. (2012). Des formateurs d'enseignants à l'épreuve d'une réforme : crise(s) et reconfigurations potentielles. Les Sciences de l'Éducation pour l'Ère Nouvelle, 45(3), 39-63. 
Piret, A., Nizet, B., \& Bourgeois, E. (1996). L'analyse structurale, une méthode d'analyse de contenu pour les sciences humaines. Paris-Bruxelles : De Boeck.

Rey, B. (2006). Les compétences professionnelles et le curriculum : des réalités conciliables ? In Y. Lenoir \& M.-H. Bouillier-Oudot (Eds.), Savoirs professionnels et curriculum de formation (p. 83-107). Québec : Les Presses de l'université Laval.

Schön, D-A. (1994). Le praticien réflexif : à la recherche du savoir caché dans l'agir professionnel (Trad J. Heynemand \&\& D. Gagnon). Montréal : Éditions logiques (première édition 1983).

Stroobants, M. (2002). La qualification ou comment s'en débarrasser. In J. Dolz \& E. Ollagnier (Eds.). L'énigme de la compétence en éducation, Bruxelles : De Boeck Université.

Teisseire, X., Rouvière, F., Boussagol, B., \& Perez-Roux, T. (2018). Le Groupe d'Entrainement à l'Analyse des Pratiques en Rééducation : une innovation pédagogique pour la professionnalisation des étudiants en kinésithérapie. Kinésithérapie, la revue, 18(193), 21-26.

Tremblay, G. (1990). À propos des compétences comme principe d'organisation d'une formation. Éducation Permanente, 103(2), 77-103.

Wittorski, R. (1998). De la fabrication des compétences. Éducation Permanente, 135, 57-69.

Wittorski R. (2003) (Ed.). Analyse de pratiques et professionnalisation des enseignants. CNAM, Centre de Recherche sur la Formation.

Wittorski, R. (2008). Professionnaliser la formation : enjeux, modalités, difficultés. Formation Emploi, 101, 105-117.

\section{NOTES}

1. Le terme de dispositif de formation est à comprendre ici dans une dimension macro de "système de formation ", comme un produit de l'institution qui définit un modèle de formation inséparable d'un nouveau modèle de l'action (Demaizière, 2008) présentant ses finalités et sa planification des contenus.

2. Notre approche s'appuie sur la sociologie de la traduction. Des travaux issus de l'analyse de l'activité ont par ailleurs discuté des référentiels de compétences, en particulier Balas (2016).

3. Cursus «Licence Master Doctorat » harmonisant les systèmes d'études supérieures européens dans le cadre du processus de Bologne (2010).

4. Ce qui est, dans ce référentiel, nommé « activités » correspond plutôt à des tâches, au sens que la psychologie ergonomique a donné à ce terme, c'est à dire au travail prescrit et non au travail réel.

5. Arrêté du 2 septembre 2015 relatif au diplôme d'État de masseur-kinésithérapeute. BO SantéProtection sociale - Solidarité, $n^{\circ}$ 2015/8 du 15 septembre, p. 275

6. Arrêté du 2 septembre 2015 relatif au diplôme d'État de masseur-kinésithérapeute. BO SantéProtection sociale - Solidarité, $n^{\circ}$ 2015/8 du 15 septembre, p. 275

7. Le terme de «formés » désigne les étudiants se destinant à exercer l'activité professionnelle pour laquelle ils sont en formation initiale.

8. L'autoconfontation désigne ici le visionnage par l'étudiant du film enregistré des actions qu'il a réalisées lors de la situation simulée. L'enseignant pointe alors les éléments de la pratique de l'étudiant qui lui apparaissent dangereux ou néfastes pour le patient et dont l'étudiant n'a pas encore pris conscience. 
9. La posture peut être définie comme «la manifestation (physique ou symbolique) d'un état mental. Façonnée par nos croyances et orientée par nos intentions, elle exerce une influence directrice et dynamique sur nos actions, leur donnant sens et justification » (Lameul, 2008, p. 89). 10. Personnels administratifs

\section{RÉSUMÉS}

Cet article vise à comprendre, à l'échelle d'un institut de formation en masso-kinésithérapie, le travail de traduction d'une équipe de direction, d'adaptation de ses formateurs et des tuteurs de stage confrontés à la mise en œuvre de la réforme des études de 2015. Le contexte institutionnel présenté à travers une analyse comparative des textes réglementant la formation montre le passage d'une approche centrée sur les contenus à une approche par les compétences dans un mouvement d'universitarisation et de professionnalisation des études. L'assise théorique de l'étude développe les notions de professionnalisation et d'universitarisation. La démarche de recherche s'appuie sur la sociologie de la traduction et une analyse qualitative de données (observations, focus group, entretiens) recueillies les deux premières années de mise en œuvre de la réforme. Les résultats mettent en relief trois niveaux du processus orientant le travail des acteurs. À un niveau macro, la traduction de la réforme montre une asynchronie dans l'espace touchant d'abord la direction, puis les formateurs proches de celle-ci et enfin les tuteurs de stage sur les lieux de travail. À un niveau méso, les tensions chez les formateurs révèlent un mouvement d'universitarisation-professionnalisation ambivalent, entre la satisfaction de la théorisation de leurs savoirs par la recherche et la crainte d'une minoration de la transmission des gestes du métier. Enfin, à un niveau micro, la focalisation sur les formateurs met en lumière leur professionnalisation secondaire, après celle de masseur-kinésithérapeute, rendue nécessaire par les orientations de la réforme.

This paper aims to understand, at the scale of a physiotherapy training institute, the management team's work and that of its trainers and training tutors faced with the implementation of the 2015 studies reform. The institutional context is presented through a comparative analysis of the national physiotherapy training texts. It shows the shift from a content-centered approach to a skills-based approach related to a dual process of universitarizing and professionalizing of studies. The theoretical background develops the notions of one's professionalization and universitarization. The research approach is based on the actor-network theory and on a qualitative data analysis (observations, focus groups, interviews) collected during the first two years of the implemented reform. The results highlight three key aspects of the process which have guided the stakeholders involved. At a macro level, the implementation of this reform shows an asynchrony affecting first the managers then the trainers close to them in the institute and finally the training tutors in the workplace. At a meso level, the tensions among the trainers reveal an ambivalent universitarizationprofessionalization movement. This goes from the satisfaction of theorizing their physiotherapeutic knowledge through research to the fear of a reduction in the transmission of professional gestures. Finally, at a micro level, the focus on trainers highlights their secondary professionalization, after that of physiotherapist, made necessary by the new approaches taken by the reform. 
INDEX

Keywords : training, professionalization, universitarization, physiotherapy, actor-network Mots-clés : formation, professionnalisation, universitarisation, masso-kinésithérapie, traduction

\section{AUTEURS}

\section{ÉRIC MALEYROT}

LIRDEF - Université Paul Valéry Montpellier 3, Route de Mende, 34199 Montpellier Cedex 5 eric.maleyrot@univ-montp3.fr

\section{THÉRÈSE PEREZ-ROUX}

LIRDEF - Université Paul Valéry Montpellier 3, Route de Mende, 34199 Montpellier Cedex 5 therese.perez-roux@univ-montp3.fr

\section{CHARLOTTE POURCELOT}

LIRDEF - Université Paul Valéry Montpellier 3, Route de Mende, 34199 Montpellier Cedex 5 charlotte.pourcelot@univ-montp3.fr

\section{PIERRE HÉBRARD}

LIRDEF - Université Paul Valéry Montpellier 3, Route de Mende, 34199 Montpellier Cedex 5 pierhebrard@orange.fr 